\title{
Traction with snare during endoscopic submucosal dissection of a gastrointestinal stromal tumor in the gastric fundus
}
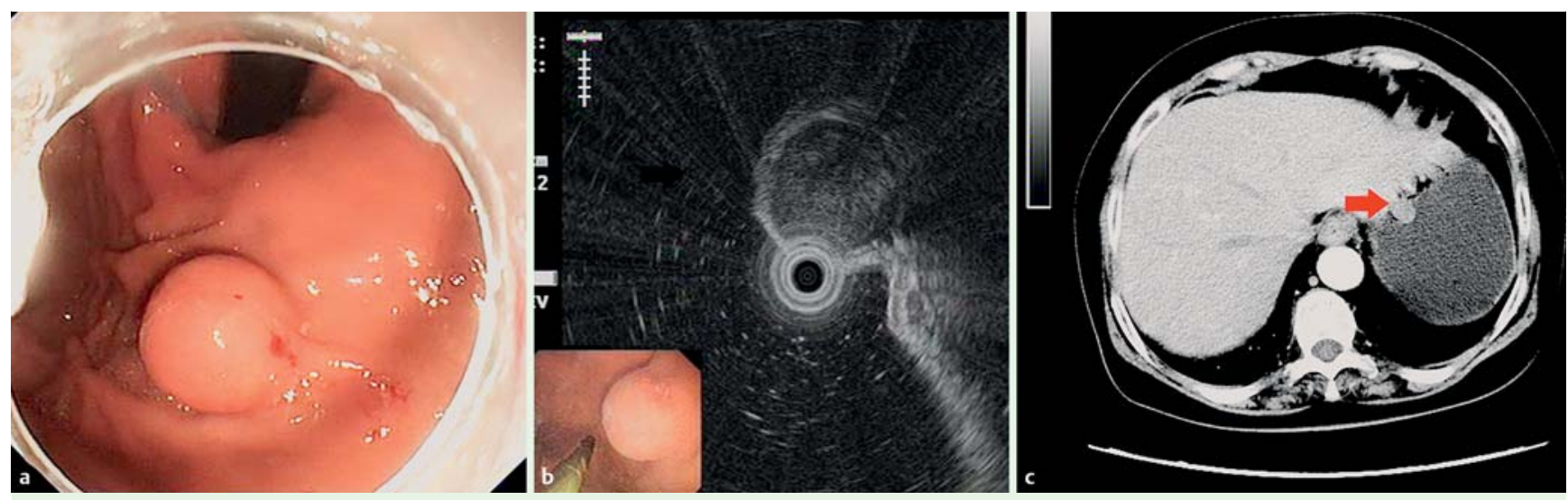

Fig. 1 Endoscopic features of a gastrointestinal stromal tumor (GIST) in the stomach. a Conventional endoscopic image of the GIST in the gastric fundus. b Endosonographic image of the lesion located in the submucosal layer. c Abdominal enhanced computed tomographic (CT) view. No extraluminal growth was identified (arrow).
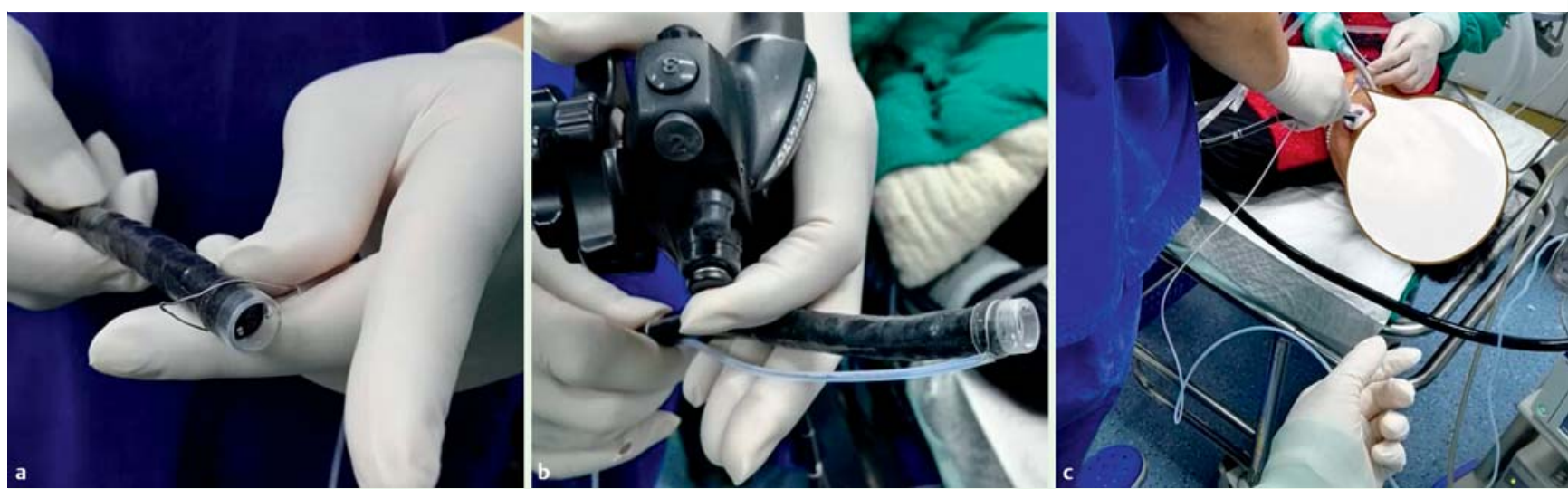

Fig.2 A snare with a lockable handle is used as a "second hand" during ESD. a, b The snare with a lockable handle is tightened on the endoscope behind the distal edge of the attached transparent hood. c A separate assistant maneuvers the snare externally to provide adequate traction in the optimal direction.

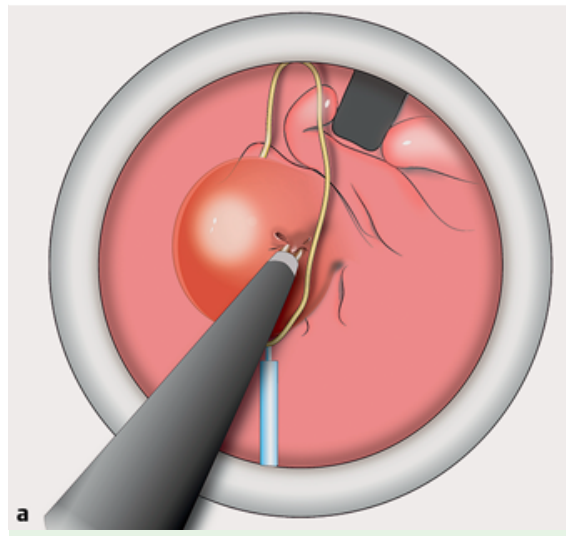

b

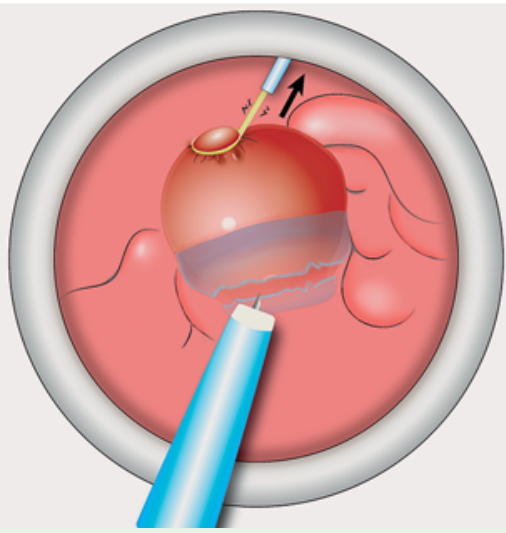

Fig. 3 Schema of the procedure. a A hemostatic forceps was used to nip the top of the lesion. The snare is guide over the forceps to grasp the submucosal tumor. b During endoscopic submucosal dissection (ESD) the snare is maneuvered externally to provide adequate traction in the optimal direction.
A clear view is necessary for safe and effective endoscopic submucosal dissection (ESD) [1]. Recently, the dental floss and endoclip method has been reported to improve visualization of the submucosal layer [2]. However, the dental floss may injure the surrounding tissue by mechanical cutting. Second, the anchorage of the target lesions can sometimes be easily dislodged. Third, conventional endoclips allow only one opportunity to grasp the tissue, and thus a new endoclip would be needed when anchoring was dislodged or inappropriately applied, which would waste time. To overcome these issues, we have designed a novel method, using a snare to help provide traction during ESD 


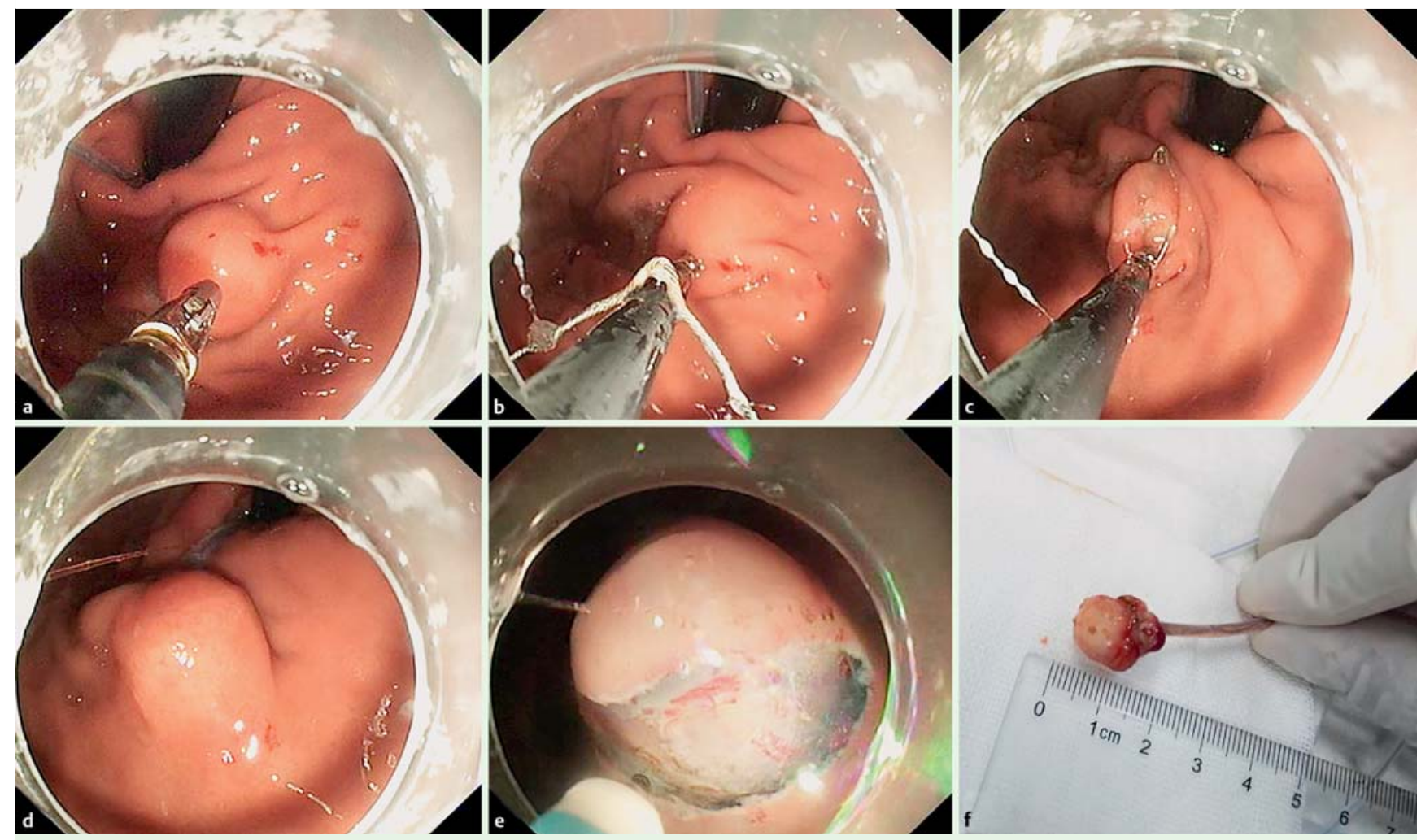

Fig. 4 Traction with a snare during endoscopic submucosal dissection (ESD) for a gastrointestinal stromal tumor (GIST) in the gastric fundus. a - c A hemostatic forceps that nips the top of the lesion provides a guide for the snare to grasp the submucosal tumor. $\mathbf{d}$ The snare provided adequate tension in the optimal direction when maneuvered externally by the assistant. e Snare traction-assisted ESD was performed for a fully circumferential lesion. $\mathbf{f}$ The resected specimen was retrieved directly using the snare.
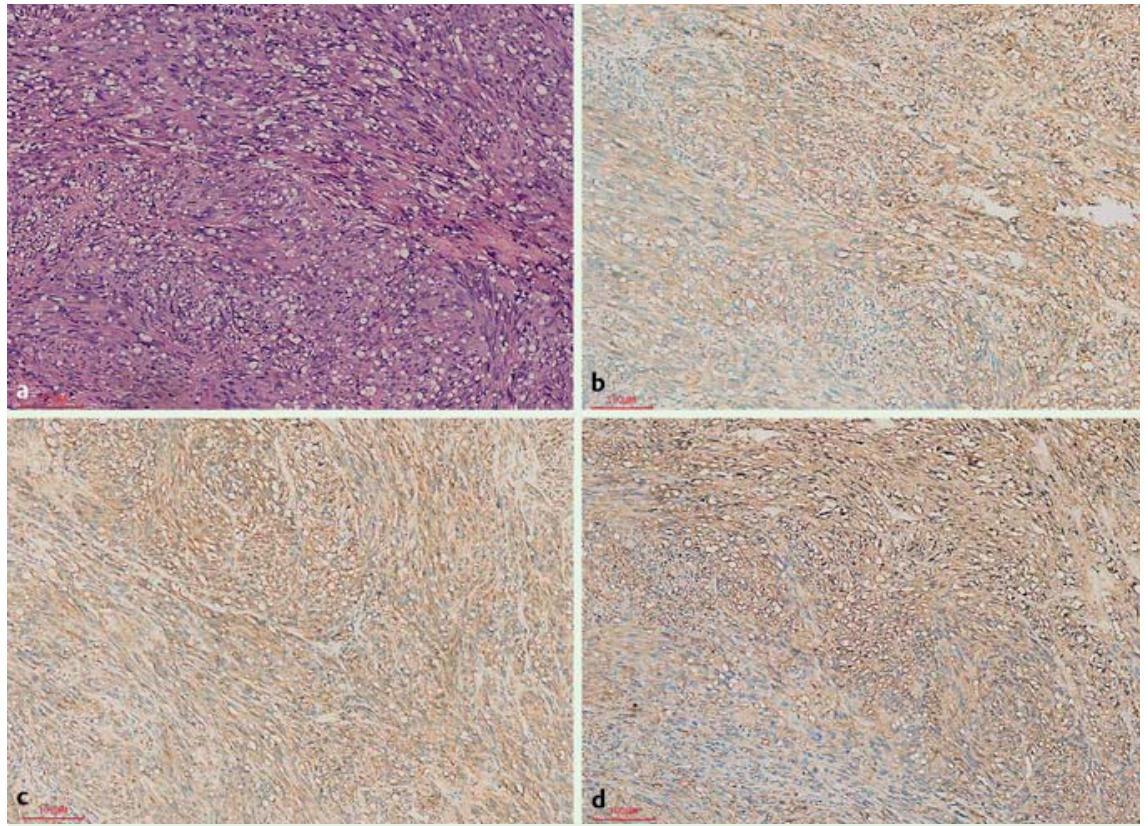

Fig. 5 Histological findings identifying the gastrointestinal stromal tumor (GIST), with hematoxylin \& eosin (H\&E) and immunohistochemical staining (bars, $100 \mu \mathrm{m})$. a The gastric GIST displays spindle cell type (H\&E); and is positive for: $\mathbf{b}$ DOG1; c CD117; d CD34.

of a gastrointestinal stromal tumor (GIST) in the gastric fundus.

A 64-year-old woman presented to our hospital with chronic persistent upper ab-
Endoscopic ultrasonography revealed a hypoechoic mass about $1.5 \times 1.6 \mathrm{~cm}$ in size located in the submucosal layer ( $\bullet$ Fig.1b). No extraluminal growth was identified on abdominal enhanced computed tomography (CT) ( $\bullet$ Fig.1c). A commercially available snare with a lockable handle (SD-230U-20; Olympus, Tokyo, Japan) was tightened around the endoscope (GIF-260Z; Olympus) behind the distal edge of the attached transparent hood before insertion ( $\bullet$ Fig. 2 a, - Fig.2b). After the tumor had been reached, a hemostatic forceps (FD-410LR; Olympus) was used to nip the top of the lesion. This provided a guide over which the snare was led to grasp the submucosal tumor ( $\bullet$ Fig.3a). This facilitated anchorage of the target lesion at a single point, at which a more uniform and firmer traction force could be applied ( $\bullet$ Fig. 3 b). The forceps were removed after the submucosal tumor had been grasped. Thus the angle of the grasped lesion could be precisely adjusted in any direction as needed for a clear view during dissection, by a separate assistant who pulled or pushed the snare ( Fig. 2c).

The total procedure took less than approximately 5 minutes from grasping the 


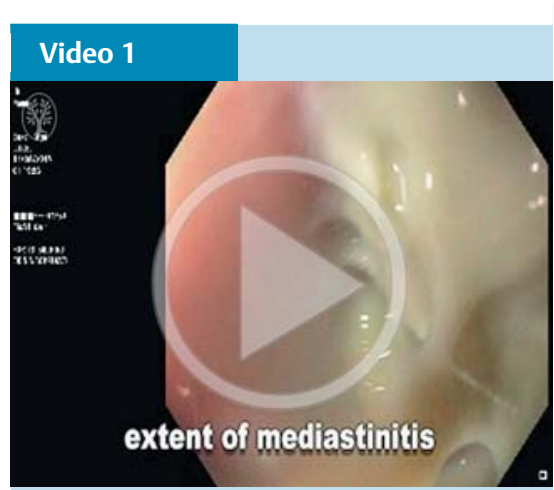

Use of a snare to provide traction during endoscopic submucosal dissection of a gastrointestinal tumor (GIST) in the gastric fundus.

submucosal tumor until the final dissection ( Fig.4a, $\odot$ Fig.4b, Fig.4c, $\odot$ Fig.4d, $\odot$ Fig.4e, $\odot$ Video 1). Notably, if there had been perforation during ESD, the tumor, because it was grasped by the snare, would not have dropped into the abdominal cavity. The defect was completely closed with 5 endoclips (Olympus HX-610-135) and no symptoms associated with peritonitis appeared thereafter. Generally, the ESG-400 generator was used for hemostasis (40W, Spray Coag setting; Olympus). The resected specimen could be removed directly and separately with the snare without retrieving the scope ( Fig.4f). Histologically, the submucosal tumor was diagnosed as a
GIST (low grade malignancy, and positive for CD117, CD34, DOG1) with hematoxylin \& eosin staining and associated immunostaining ( $\bullet$ Fig.5).

This technique also has the advantage that the snare can be released in order to regrasp another part of the lesion as needed. Limitations of this technique are that coordination between endoscopist and assistant is needed when releasing and re-applying the snare, and it is more difficult to use this method in a limited working space. This method could be applied for both epithelial and subepithelial tumors; however when it is used for early cancers, the tumor must be grasped more gently to avoid damaging the lesion and thus hampering subsequent histological diagnosis.

Endoscopy_UCTN_Code_TTT_1AO_2AG

Competing interests: None

\section{Mu Han Lü ${ }^{1}$, Kuang-I Fu' ${ }^{1,2}$, Zhong Qiong Wang', Yong Liư ${ }^{3}$, Hong Fen Xia ${ }^{4}$, Ming-Ming Deng ${ }^{1}$}

${ }^{1}$ Department of Gastroenterology, Affiliated Hospital of Luzhou Medical College, Luzhou, China

2 Department of Endoscopy, Kanma

Memorial Hospital, Tokyo, Japan
${ }^{3}$ Department of Pathology, Affiliated Hospital of Luzhou Medical College, Luzhou, China

${ }^{4}$ Endoscopy Center, Affiliated Hospital of Luzhou Medical College, Luzhou, China

\section{References}

1 Imai $K$, Hotta K, Yamfaguchi $Y$ et al. Safety and efficacy of endoscopic submucosal dissection of rectal tumors extending to the dentate line. Endoscopy 2015; 47: 529-532

2 Suzuki S, Gotoda T, Kobayashi Y et al. Usefulness of a traction method using dental floss and a hemoclip for gastric endoscopic submucosal dissection: a propensity score matching analysis (with videos). Gastrointest Endosc 2016; 83: 337-346

Bibliography

DOI http://dx.doi.org/

10.1055/s-0042-105644

Endoscopy 2016; 48: E183-E185

(c) Georg Thieme Verlag KG

Stuttgart · New York

ISSN 0013-726X

Corresponding author

Ming-Ming Deng, MD

Department of Gastroenterology

Luzhou Medical College Affiliated Hospital

Luzhou 646000

P.R. China

Fax: +86-830-3165331

563136441@qq.com 